Vietnam Journal of Mechanics, VAST, Vol.41, No. 1 (2019), pp. 89-103

DOI: https://doi.org/10.15625/0866-7136/13018

\title{
INVESTIGATION OF AERODYNAMICS AND LONGITUDINAL STABILITY OF UNMANNED AERIAL VEHICLE WITH ELEVATOR DEFLECTION
}

\author{
Hoang Thi Bich Ngoc*, Bui Vinh Binh \\ Hanoi University of Science and Technology, Vietnam \\ *E-mail: ngoc.hoangthibich@hust.edu.vn
}

Received: 28 August 2018 / Published online: 28 February 2018

\begin{abstract}
The elevator is usually hinged to the horizontal tail, which acts as a balance and controls the altitude, establishes a steady motion for the aircraft at all lift coefficients. During elevator rotating, the aircraft needs to be stable to establish a new altitude. The horizontal tail has a major role in the value of the airplane's pitching moment (due to the long arm from the aerodynamic center of the tail to the center of gravity) for the equilibrium and stability of the aircraft. The horizontal tail should be considered as an aerodynamic component behind the main wing, influenced by the wing downwash wing rather than just a minor wing. Therefore, the aim of this study is to examine the flow through unmanned aerial vehicles (UAV) including the main wing, tail and body and to calculate the aerodynamic force on the horizontal tail when rotating the elevator using the Fluent software for the viscous flows. Small disturbance theory was used to calculate the longitudinal stability of the UAV when controlling the elevator. Flying qualities are assessed to show that changes in the aerodynamic characteristics of the wing, tail, fuselage and configuration of the UAV may be required.
\end{abstract}

Keywords: UAV aerodynamics; horizontal tail and elavator; equilibrium; longlitudinal stability.

\section{INTRODUCTION}

When flying, the aircraft should be balanced and stable with environmental impact using necessary controls. The horizontal tail with the elevator ensures the balance of the aircraft with all variations in lift coefficient. In calculating aerodynamic forces, viewing the horizontal tail like a miniature main wing will eventually lead to large errors. The horizontal tail should be placed in the main wing wake and be influenced by the downwash effect of the wing [1,2]. That is, the incidence velocity to the horizontal tail is not the velocity at infinity as for the main wing. Therefore, the aim of this study is to examine the flow through the aircraft including the main wing, tail and body and to calculate the aerodynamic force on the horizontal tail when rotating the elevator using the Fluent

(C) 2019 Vietnam Academy of Science and Technology 
software for the viscous flows. When the elevator angle is zero, the aircraft is in equilibrium flight. When the elevator is deflected, it changes the lift on the horizontal tail and the aircraft nose moves up or down. Until the aircraft reaches a certain altitude, the elevator angle returns to zero and the aircraft registers equilibrium. Such changes in lift and pitching moment require consideration of the longitudinal dynamic stability of the aircraft.

By experimental method, Thomas and Wonhart [3] determined the lift coefficient, drag coefficient and moment coefficient of a model airplane to determine the static stability of the airplane. The numerical study has used the size and configuration of the experimental model airplane in [3] to calculate and to perform comparisons of numerical results with experimental results, this allows applying calculations for UAV. Our computer program for longitudinal stability calculations is validated when calculating the stability of the model of Navion aircraft given in [4,5]. Flight quality assessment may lead to requirements for changes in aerodynamic design and configuration of aircraft $[6,7]$.

\section{AERODYNAMIC COEFFICIENTS AND PITCHING MOMENT COEFFICIENTS}

\subsection{Aerodynamic model of UAV}

Fig. 1 is the configuration and size of the UAV considered in this study. Dimensions of the fuselage are given in Tab. 1. Dimensions of the main wing and horizontal tail are shown in Tab. 2. The vertical tail is arranged at the two tips of the horizontal and its profile is Naca 0010 . The UAV velocity is $44.4 \mathrm{~m} / \mathrm{s}$.
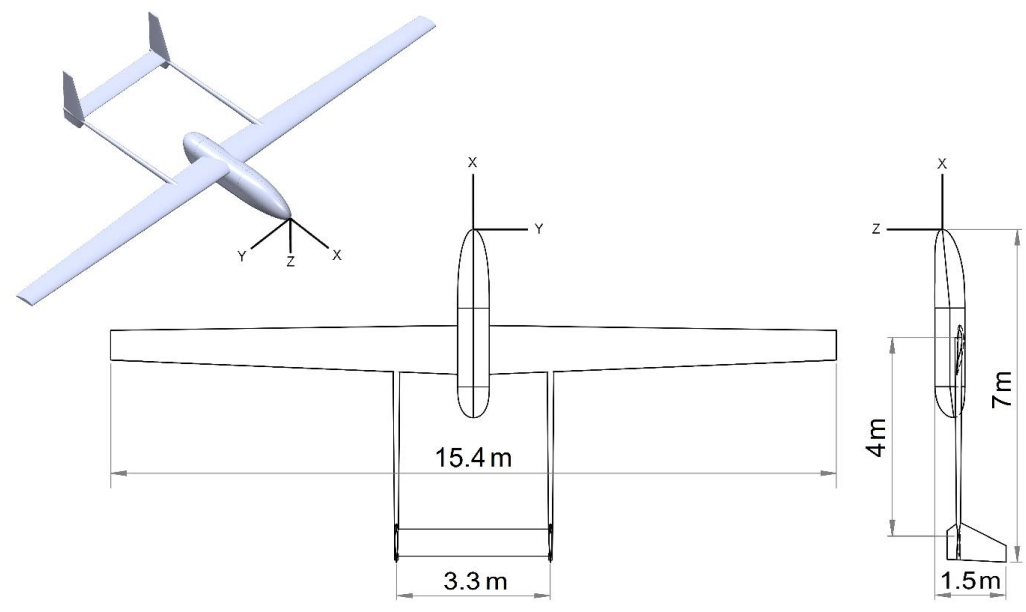

Fig. 1. Configuration and size of UAV

For the boundary of meshing (Fig. 2), the inlet and outlet surfaces were respectively, $20 \bar{c}_{w}$ and $50 \bar{c}_{w}$ far away from the UAV ( $\bar{c}_{w}$ is the mean chord of the wing); the top and bottom surfaces were $20 \bar{c}_{w}$ far away from the UAV; the side surface was $0.5 b_{w}\left(b_{w}\right.$ is the wingspan) far away from the UAV; For boundary conditions, the symmetry condition was at the symmetry surface; the velocity at infinity $\left(V_{\infty}\right)$ was at the inlet surface and the 
Table 1. The fuselage coordinates $(\mathrm{mm})$

\begin{tabular}{rrrrrrrr}
\hline$x$ & $y$, lower & $y$, upper & \multicolumn{1}{c}{$z$} & $x$ & $y$, lower & $y$, upper & $z$ \\
\hline & -158 & -158 & 0 & 1660 & -318 & 318 & 335 \\
130 & -268 & 21 & 150 & 3320 & -318 & 318 & 335 \\
280 & -300 & 104 & 210 & 3720 & -234 & 285 & 286 \\
520 & -318 & 197 & 270 & 4000 & 84 & 84 & 0 \\
\hline
\end{tabular}

Table 2. Geometry and dimensions of the main wing and horizontal tail

\begin{tabular}{lccc}
\hline \multicolumn{1}{c}{ Parameter } & Symbol & Main wing & Horizontal tail \\
\hline Profile & Naca & 4412 & 0010 \\
Span $(\mathrm{m})$ & $b$ & 15.4 & 3.3 \\
Root chord $(\mathrm{m})$ & $c_{r}$ & 1.0 & 0.57 \\
Tip chord $(\mathrm{m})$ & $c_{t}$ & 0.7 & 0.57 \\
Mean chord $(\mathrm{m})$ & $\bar{c}$ & 0.859 & 0.57 \\
Aspect ratio & $A_{R}$ & 17.6 & 5.0 \\
Setting angle (degree) & $i$ & 4 & 0 \\
Sweep angle of leading edge (degree) & $\Lambda_{L E}$ & 0.57 & 0 \\
Sweep angle of trailing edge (degree) & $\Lambda_{T E}$ & 1.72 & 0 \\
Area $\left(\mathrm{m}^{2}\right)$ & $S$ & 12.94 & 1.80 \\
\hline
\end{tabular}

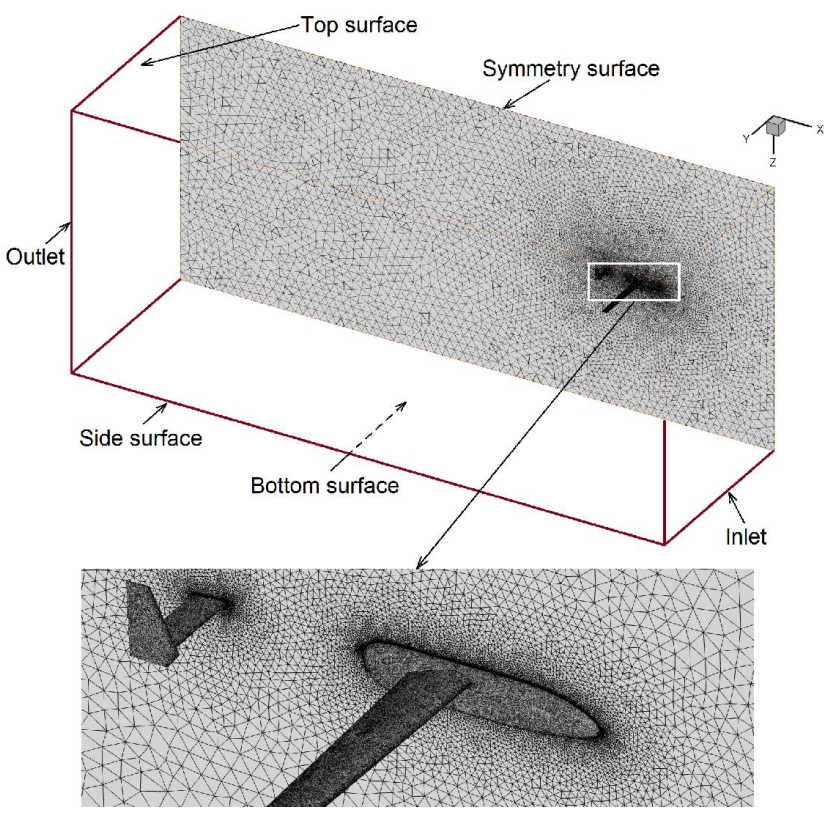

Fig. 2. Boundary of meshing and grid on the symmetry surface of UAV 
pressure at infinity $\left(p_{\infty}\right)$ was at the outlet surface; the symmetry condition was at the side, top and bottom surfaces; the no-slip boundary condition $(\vec{v}=0)$ was enforced at wall (of the UAV) by default [8]. The grid size was fine enough in boundary layers, wing tip zone, intersection domains of UAV components $[9,10]$. Model of turbulence $(k-\varepsilon)$ was used for all simulation problems using Fluent 6.3 software in this work. Operations and meshing techniques with Fluent are verified in Section 2.2 by the comparison of numerical results with experimental results on a model aircraft [3].

Fig. 3 presents streamlines through wing and fuselage with angles of attack $\alpha_{\mathrm{UAV}}=$ $0^{\circ}$ and $\alpha_{\mathrm{UAV}}=14^{\circ}$. In case of $\alpha_{\mathrm{UAV}}=0^{\circ}$, streamlines were smooth, which showed that there was no separation on the wing upper surface and at the trailing edge there was no vortex (except the vortex at the wing tip caused by circular flows from the lower to the upper surfaces of the wing). In case of $\alpha_{\mathrm{UAV}}=14^{\circ}$, streamlines were no smooth on the wing and fuselage. This meant that separations took place on the wing upper surface and form vertices at the wing rear.

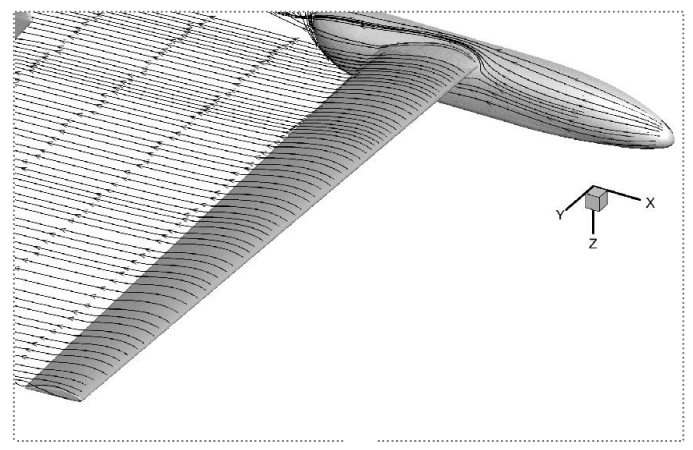

(a) $\alpha_{\mathrm{UAV}}=0^{\circ}$

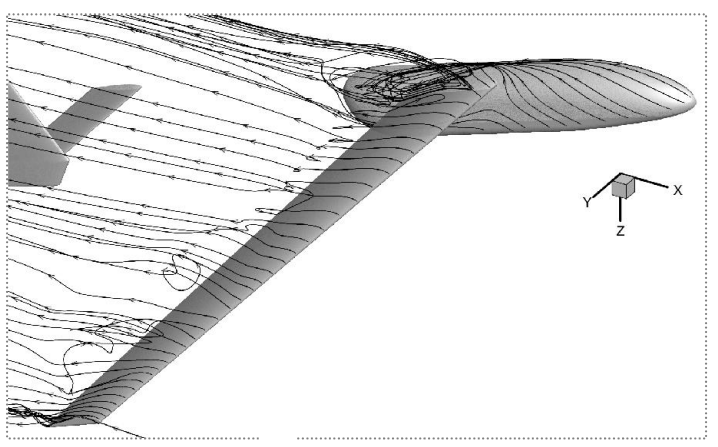

(b) $\alpha_{\mathrm{UAV}}=14^{\circ}$

Fig. 3. Streamlines through wing and fuselage

Fig. 4 shows the lift and drag coefficients with respect to the angle of attack $\alpha_{\mathrm{UAV}}$ for the UAV, main wing, horizontal tail and fuselage when the elevator deflection angle was zero $\left(\delta_{e}=0^{\circ}\right)$. It was observed that the lift and drag coefficients of the UAV were mainly due to the main wing. The lift coefficient of the fuselage was small, but its drag coefficient was equivalent to that of the horizontal tail. Lift and drag of the vertical tail were not significant (not shown in Fig. 4). The lift of the horizontal tail was very small compared to that of the main wing, but had a great influence on the balance and longitudinal stability of the UAV. This was because the large distance (arm) from the aerodynamic center of the horizontal tail to the gravity center of the UAV, thus creating a great pitching moment. Lift coefficients of horizontal tail were negative with angles of attack less than 2 degrees $\left(\alpha_{\mathrm{UAV}}<2^{\circ}\right)$. At angle of attack $\alpha_{\mathrm{UAV}}=0^{\circ}$, lift coefficient of the horizontal tail $C_{L(H)}=$ -0.015 that was due to downwash effect of the main wing (because the horizontal tail had a zero setting angle and symmetry profile).

Extracting from Fig. 4 graphs of lift and drag coefficients of the horizontal tail (as a component of the UAV) that are shown in Fig. 5 (with $\delta_{e}=0^{\circ}$ ). Fig. 5 also shows graphs 


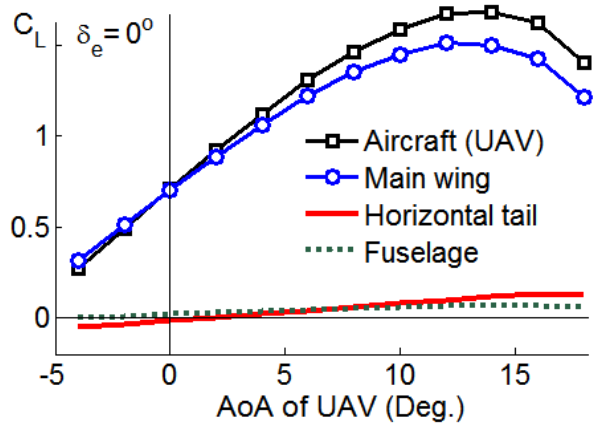

(a) Lift coefficient

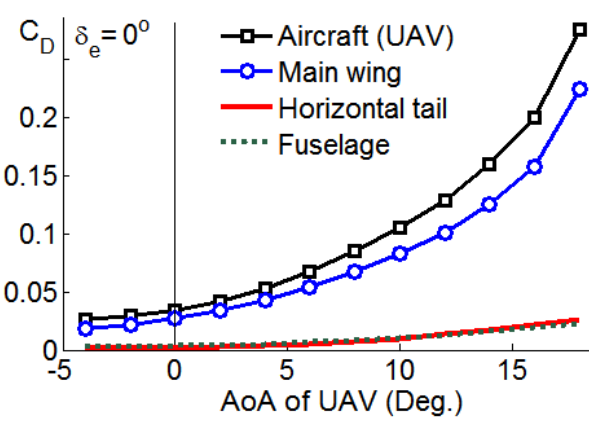

(b) Drag coefficient

Fig. 4. Aerodynamic coefficients of the UAV, main wing, horizontal tail and fuselage

of lift and drag coefficients of the horizontal tail (which is a component of the UAV) with elevator deflection angles $\delta_{e}=10^{\circ}$ and $\delta_{e}=-5^{\circ}$. With three values of the elevator deflection angle $\left(\delta_{e}=0^{\circ}, \delta_{e}=10^{\circ}, \delta_{e}=-5^{\circ}\right)$, lift coefficients of the horizontal tail at angle of attack $\alpha_{\mathrm{UAV}}=0^{\circ}$ are respectively $-0.015,0.062,-0.054$ (as shown in Tab. 3).

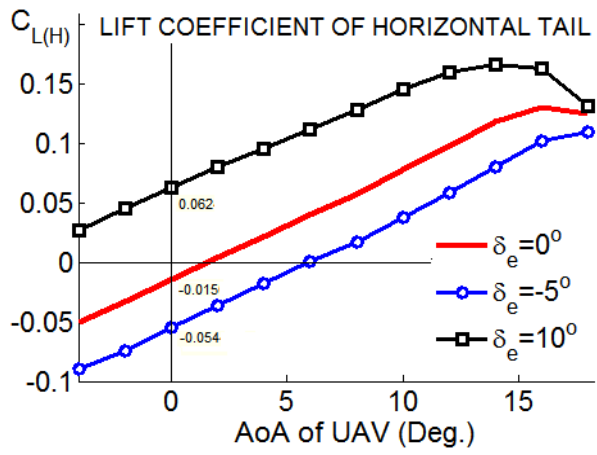

(a) Lift coefficient

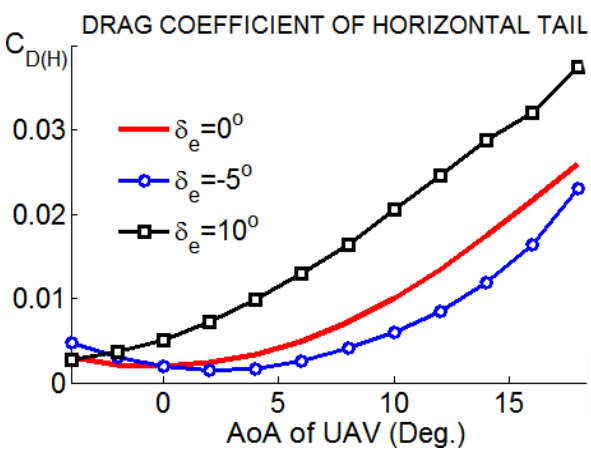

(b) Drag coefficient

Fig. 5. Aerodynamic coefficients of the UAV's horizontal tail

Fig. 6 is the 3D distribution of pressure coefficients on a half of the tail alone when the elevator deflection angle at three values of $\delta_{e}\left(0^{\circ}, 10^{\circ},-5^{\circ}\right)$. In the case of $\delta_{e}=0^{\circ}$, pressure coefficients on the upper and lower surfaces of the tail were the same, so lift coefficient of the tail was zero $\left(C_{L(H)}=0\right)$. Lift coefficients of the tail alone at three values of $\delta_{e}\left(0^{\circ}, 10^{\circ},-5^{\circ}\right)$ are shown in Tab. 3 .

Comparing the results in Tab. 3 shows that the lift coefficient of tail alone differed significantly from that of the UAV's horizontal tail when the last suffered from interaction with other components of the UAV, especially influenced by the main wing downwash [11]. Therefore, the calculation of the aerodynamic force on the horizontal tail alone causes a large error. 


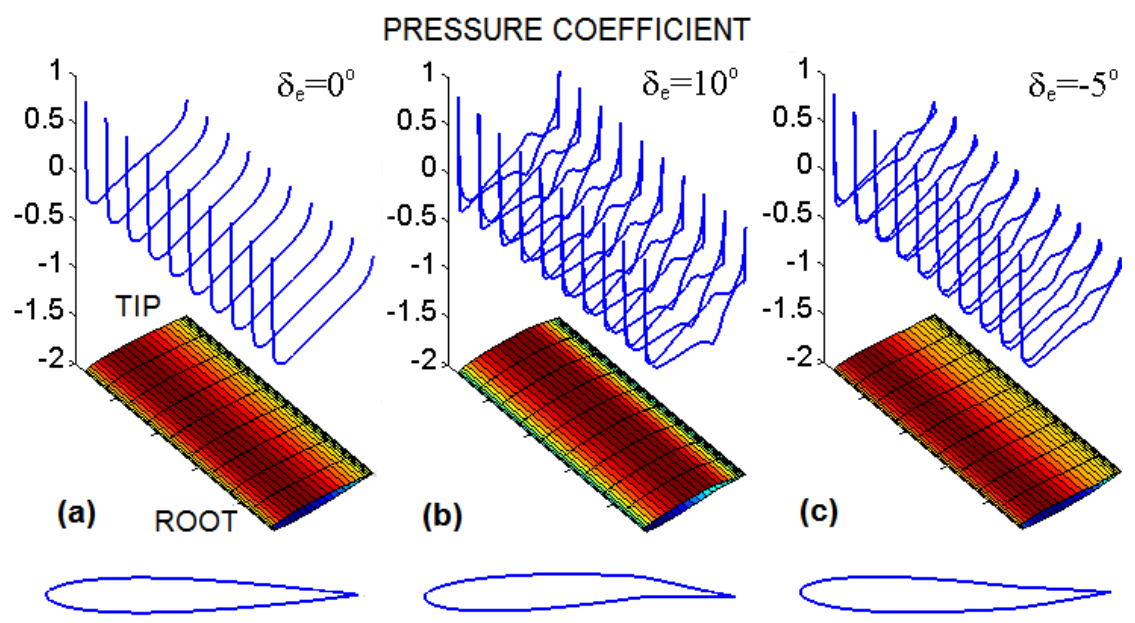

Fig. 6. 3D distribution of pressure coefficients on a half of the tail alone (a) $\delta_{e}=0^{\circ}$; (b) $\delta_{e}=10^{\circ}$; (c) $\delta_{e}=-5^{\circ}$

Table 3. Lift coefficient of Horizontal tail $C_{L(H)}$ with $\alpha_{\mathrm{UAV}}=0^{\circ}$

\begin{tabular}{cccc}
\hline Elevator angle & $\delta_{e}=0^{\circ}$ & $\delta_{e}=10^{\circ}$ & $\delta_{e}=-5^{\circ}$ \\
\hline UAV's tail & -0.015 & 0.062 & -0.054 \\
Tail alone & 0 & 0.15 & -0.1 \\
\hline
\end{tabular}

Note that the results of the aerodynamic coefficients of the horizontal tail in Fig. 4 and Tab. 3 were determined when considering the horizontal tail as a component of the aircraft (UAV). Therefore, they were referred to the main wing area of the aircraft $\left(S_{W}\right)$ with the formulas of lift coefficient $C_{L(H)}$ and drag coefficient $C_{D(H)}$ as follows

$$
C_{L(H)}=\frac{L_{H}}{0.5 \rho V_{\infty}^{2} S_{W}}, \quad C_{D(H)}=\frac{D_{H}}{0.5 \rho V_{\infty}^{2} S_{W}},
$$

where $\rho$ is the air density, $V_{\infty}$ is the velocity at infinity, $L_{H}$ and $D_{H}$ indicate the lift and drag of the horizontal tail under the main wing downwash effect.

In case of considering the horizontal tail is a lift wing alone, the aerodynamic coefficients are referred to the horizontal tail area $\left(S_{H}\right)$ as the following

$$
C_{L(H)}=\frac{L_{H}}{0.5 \rho V_{\infty}^{2} S_{H}}, \quad C_{D(H)}=\frac{D_{H}}{0.5 \rho V_{\infty}^{2} S_{H}} .
$$

If comparing the aerodynamic coefficients on the horizontal tail using $3 \mathrm{D}$ simulation method and with those calculated by semi-analytical method based on $2 \mathrm{D}$ results, it is necessary to use the formula (2). Because in the semi-analytical method, the horizontal tail is considered a wing alone subjected to a uniform velocity field $V_{\infty}$ and a downwash angle $\varepsilon$ determined by semi-analytical method (according to the 3D simulation method, the downwash angle $\varepsilon$ changes in all three directions $(x, y, z))$. 
The lift coefficient of the horizontal tail was much smaller than that of the main wing. However, the pitching moment of the horizontal tail was much larger than that of the main wing and played an important role in the balance of the aircraft. Fig. 7 shows pitching moment coefficient of the UAV and its components at elevator deflection angles $\delta_{e}=0^{\circ}$ (Fig. 7(a)) and $\delta_{e}=10^{\circ}$ (Fig. 7(b)). It was observed that pitching moment coefficient of the fuselage was very small (near zero at the angle of attack being zero). In case of $\delta_{e}=0^{\circ}$ (Fig. 7(a)), the UAV was balanced at the angle of attack $\alpha_{\mathrm{UAV}}=0^{\circ}$ with the pitching moment coefficient of the UAV being zero, $C_{m(\mathrm{UAV})}=0$ (the UAV was in equilibrium). In case of $\delta_{e}=10^{\circ}$ (Fig. 7(b)), the pitching moment coefficient of the UAV was non-zero, $C_{m(\mathrm{UAV})} \neq 0$, and negative. The UAV was then out of balance and its nose was down to reduce altitude.

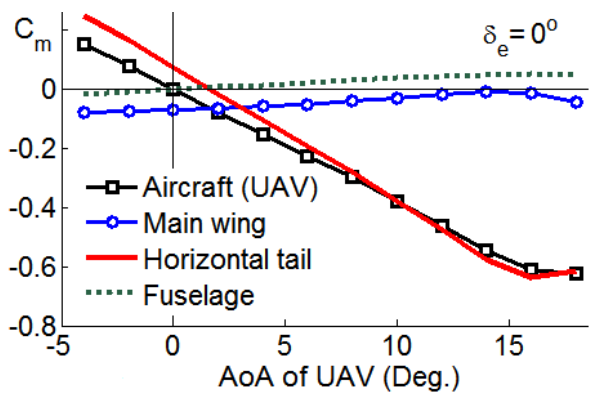

(a) $\delta_{e}=0^{\circ}$

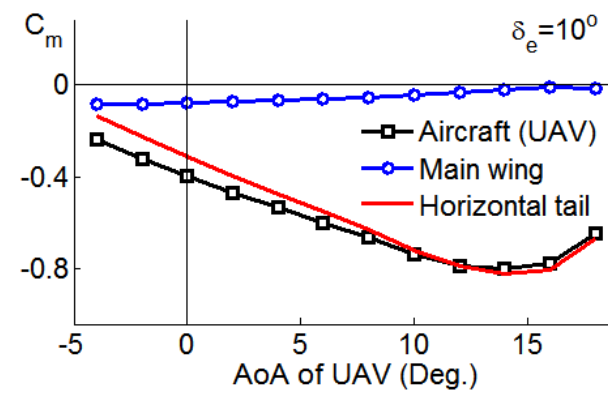

(b) $\delta_{e}=10^{\circ}$

Fig. 7. Pitching moment coefficients of the UAV, wing and horizontal tail

The longitudinal stability of the UAV when changing altitude due to elevator deflection angle control is considered in Section 3.

\subsection{Comparison of numerical and experimental results}

Fig. 8(a) presents a photograph of model airplane and its dimensions (inch units) in experiments of Thomas and Wolhart [3] with the purpose of measuring aerodynamic forces to determine the static stability of the airplane. The main wing and horizontal tail of the airplane were high swept-back, 45 degrees for the quarter lines and had the profile Naca 65A008. The main wing had the aspect ratio of 6, root chord of $233 \mathrm{~mm}$, tip chord of $140 \mathrm{~mm}$, span of $1120 \mathrm{~mm}$. The horizontal tail had the aspect ratio of 2.8 , root chord of $152 \mathrm{~mm}$, tip chord of $92 \mathrm{~mm}$, span of $340 \mathrm{~mm}$. The vertical tail had the root chord of $188 \mathrm{~mm}$, tip chord of $112 \mathrm{~mm}$, span of $211 \mathrm{~mm}$. Free Mach number $M=0.13$. Other dimensions are shown in Fig. 8(a).

From the size of the model airplane described in Fig. 8(a), the shape of the airplane was redrawn for which the grid was generated as in Fig. 8(b). Numerical results of lift and drag coefficients compared with experimental results [3] are shown in Fig. 9.

With angles of attack $\alpha=-4^{\circ} \div 14^{\circ}$, numerical and experimental results of lift coefficients were similar (differences were less than 5\%) and they were increased almost linearly. For angles of attack $\alpha=14^{\circ} \div 20^{\circ}$, lift coefficients were increased with convex 


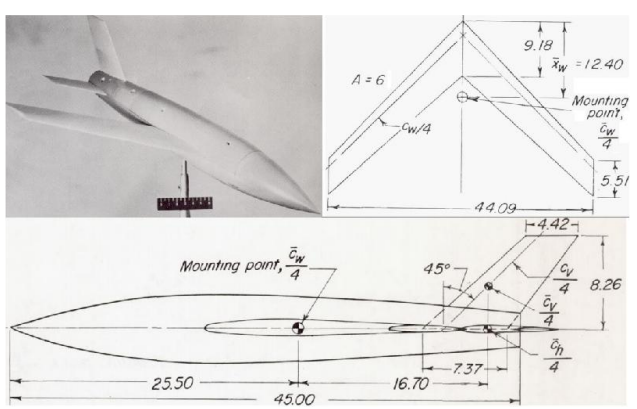

(a)

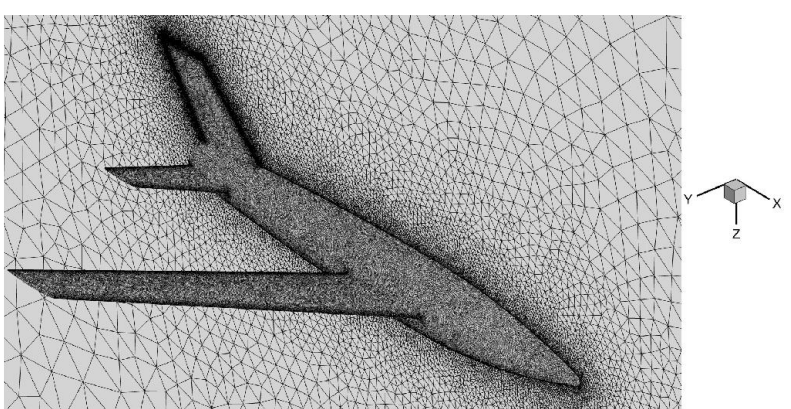

(b)

Fig. 8. (a) Dimensions of model airplane in experiment [3]; (b) Grid on the symmetrical surface of the reconstructed model airplane

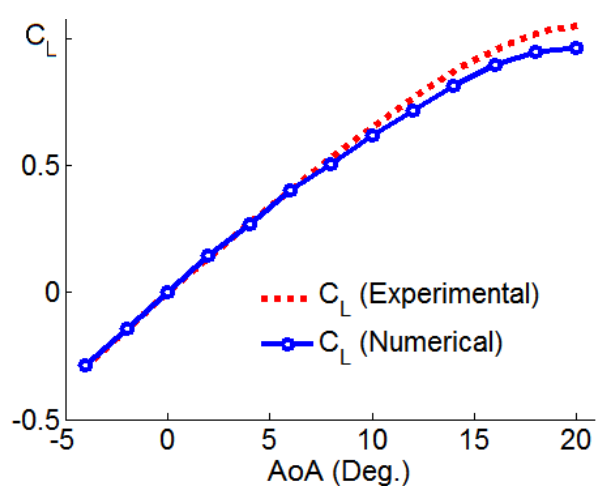

(a) Lift coefficient

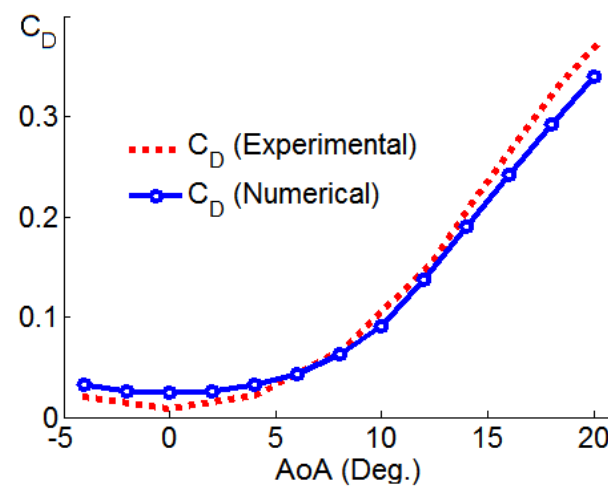

(b) Drag coefficient

Fig. 9. Aerodynamic coefficients of the airplane used in experiment [3]

curve (the speed of increasing was reduced) and differences between the numerical and experimental results were smaller than 7\% (Fig. 9(a)). Drag coefficients (Fig. 9(b)) were increased with a concave curve and had the smallest value when the angle of attack was zero degrees. Numerical and experimental results were similar with differences being less than $5 \%$ for angles of attack $\alpha=-4^{\circ} \div 14^{\circ}$. With $\alpha=14^{\circ} \div 20^{\circ}$, strong separations caused difficulties for the convergence of numerical methods; therefore, there were differences between numerical and experimental results (less than $7 \%$ ). The numerical and experimental comparisons above allow the verification of the application of Fluent software for the aerodynamic calculation of airplanes in the range of subsonic flows. 


\section{LONGITUDINAL STABILITY OF THE AIRCRAFT WITH ELEVATOR DEFLECTION}

\subsection{State equations of longitudinal motion and method of solution}

With low-speed and small-sized aircrafts (Reynolds numbers $\operatorname{Re}<10^{7}$ ), it is possible to apply small disturbance theory. The state equations of longitudinal motion of the aircraft can be represented mathematically $[4,5]$ as the following

$$
\dot{x}=A x+B \eta_{x}
$$

That is a set of first-order linear differential equations, where $x$ is the state vector $x=$ $\left(\Delta u, \Delta w, \Delta \omega_{y}, \Delta \theta\right)^{T}\left(u, v, w, \omega_{y}\right.$ are defined in Tab. $4 ; \theta$ is the pitch Euler angle; The change of parameters is denoted by the prefix $\Delta) ; \eta$ is the control vector, $\eta_{x}=\left(\Delta \delta_{e}, \Delta \delta_{T}\right)\left(\Delta \delta_{e}\right.$ is the variation in elevator deflection angle, $\Delta \delta_{T}$ is the variation in propulsive force and equals zero in this study); The matrices $A$ and $B$ contain the aircraft's aerodynamic derivatives. Rewrite Eq. (3) in matrix form as follows

$$
\begin{aligned}
{\left[\begin{array}{c}
\Delta \dot{u} \\
\Delta \dot{w} \\
\Delta \dot{q} \\
\Delta \dot{\theta}
\end{array}\right]=} & {\left[\begin{array}{cccc}
X_{u} & X_{w} & 0 & -g \\
Z_{u} & Z_{w} & u_{0} & 0 \\
M_{u}+M_{\dot{w}} Z_{u} & M_{w}+M_{\dot{w}} Z_{w} & Z_{w} M_{q}+M_{\dot{w}} u_{0} & 0 \\
0 & 0 & 1 & 0
\end{array}\right]\left[\begin{array}{c}
\Delta u \\
\Delta w \\
\Delta q \\
\Delta \theta
\end{array}\right] } \\
+ & {\left[\begin{array}{cc}
X_{\delta_{e}} & 0 \\
Z_{\delta_{e}} & 0 \\
M_{\delta_{e}}+M_{\dot{w}} Z_{\delta_{e}} & 0 \\
0 & 0
\end{array}\right]\left[\begin{array}{c}
\Delta \delta_{e} \\
0
\end{array}\right], }
\end{aligned}
$$

where the velocity, force, momentum and derivative components are defined in Tab. 4.

Table 4. Velocity, force, momentum and derivative components

\begin{tabular}{lccc}
\hline Coordinate & $x$ & $y$ & $z$ \\
\hline Angular rate & $p\left(\omega_{x}\right)$ & $q\left(\omega_{y}\right)$ & $r\left(\omega_{z}\right)$ \\
Velocity & $u$ & $v$ & $w$ \\
Force, & $X$, & $Y$ & $Z$, \\
derivative & $X_{u}=(\partial X / \partial u) / m$ & & $Z_{w}=(\partial Z / \partial w) / m$ \\
Moment, & $L\left(M_{x}\right)$, & $M\left(M_{y}\right)$, & $N\left(M_{z}\right)$ \\
derivative & $M_{u}=(\partial X / \partial u) / I_{y}$ & $M_{q}=\left(\partial M_{y} / \partial q\right) / I_{y}$ & \\
\hline
\end{tabular}

Other derivatives are defined as follows

$$
\begin{aligned}
& Z_{\delta_{e}}=\frac{\partial Z}{\partial \delta_{e}} / m, \quad M_{\delta_{e}}=\frac{\partial M_{y}}{\partial \delta_{e}} / I_{y}, \quad X_{w}=\frac{\partial X}{\partial w} / m, \quad Z_{u}=\frac{\partial Z}{\partial u} / m, \quad Z_{\alpha}=u_{0} Z_{w}, \\
& M_{w}=\frac{\partial M_{y}}{\partial w} / I_{y}, \quad M_{\dot{w}}=\frac{\partial M_{y}}{\partial \dot{w}} / I_{y}, \quad M_{\alpha}=\frac{\partial M_{y}}{\partial \alpha} / I_{y}, \quad M_{\dot{\alpha}}=\frac{\partial M_{y}}{\partial \dot{\alpha}} / I_{y} .
\end{aligned}
$$


Formulas for determining derivatives of force and moment are

$$
\begin{aligned}
& X_{u}=\frac{-\left(C_{D_{u}}+2 C_{D_{0}}\right) Q S}{m u_{0}}=C_{x_{u}} \frac{Q S}{m u_{0}}\left(\mathrm{~s}^{-1}\right), \quad X_{w}=\frac{-\left(C_{D_{\alpha}}-C_{L_{0}}\right) Q S}{m u_{0}}=C_{x_{w}} \frac{Q S}{m u_{0}}\left(\mathrm{~s}^{-1}\right), \\
& Z_{u}=\frac{-\left(C_{L_{u}}+2 C_{L_{0}}\right) Q S}{m u_{0}}=C_{z_{u}} \frac{Q S}{m u_{0}}\left(\mathrm{~s}^{-1}\right), \quad Z_{w}=\frac{-\left(C_{L_{\alpha}}+C_{D_{0}}\right) Q S}{m u_{0}}=C_{z_{w}} \frac{Q S}{m u_{0}}\left(\mathrm{~s}^{-1}\right), \\
& Z_{\alpha}=u_{0} Z_{w}\left(\mathrm{~m} / \mathrm{s}^{2}\right), \quad Z_{\delta_{e}}=-C_{z \delta_{e}} \frac{Q S}{m}\left(\mathrm{~m} / \mathrm{s}^{2}\right), \quad M_{u}=C_{m_{u}} \frac{Q S \bar{c}}{u_{0} I_{y}}(1 / \mathrm{ms}), \\
& M_{w}=C_{m_{\alpha}} \frac{Q S \bar{c}}{u_{0} I_{y}}(1 / \mathrm{ms}), \quad M_{\dot{w}}=C_{m_{\alpha}} \frac{\bar{c}}{2 u_{0}} \frac{Q S \bar{c}}{u_{0} I_{y}}\left(\mathrm{~m}^{-1}\right), \quad M_{\alpha}=u_{0} M_{w}\left(\mathrm{~s}^{-2}\right), \\
& M_{\dot{\alpha}}=u_{0} M_{\dot{w}}\left(\mathrm{~s}^{-1}\right), \quad M_{q}=C_{m_{q}} \frac{\bar{c}}{2 u_{0}} \frac{Q S \bar{c}}{I_{y}}\left(\mathrm{~s}^{-1}\right), \quad M_{\delta_{e}}=C_{m \delta_{e}} \frac{Q S \bar{c}}{I_{y}}\left(\mathrm{~s}^{-2}\right) .
\end{aligned}
$$

Longitudinal stability coefficients for $X, Z$ and $M_{y}$ are

$$
\begin{gathered}
C_{X u}=-\left(C_{D_{u}}+2 C_{D_{0}}\right), \quad C_{Z_{u}}=-\frac{M^{2}}{1-M^{2}} C_{L_{0}}-2 C_{L_{0}} \\
C_{Z_{\alpha}}=-\left(C_{L_{\alpha}}+C_{D_{0}}\right), \quad C_{Z_{\dot{\alpha}}}=-2 \eta C_{L_{\alpha_{t}}} V_{H} \frac{d \varepsilon}{d \alpha}, \quad C_{Z_{q}}=-2 \eta C_{L_{\alpha t}} V_{H} \\
C_{Z_{\delta e}}=-C_{L \delta_{e}}=-\frac{S_{t}}{S} \eta \frac{d C_{L_{t}}}{d \delta_{e}}=-\frac{S_{t}}{S} \eta \tau C_{L_{\alpha t}} \\
C_{m \alpha}=C_{L \alpha_{w}}\left(\frac{x_{c g}}{\bar{c}}-\frac{x_{a c}}{\bar{c}}\right)+C_{m \alpha f}-\eta V_{H} C_{L_{\alpha t}}\left(1-\frac{d \varepsilon}{d \alpha}\right), \quad C_{m_{\dot{\alpha}}}=-2 \eta C_{L_{\alpha t}} V_{H} \frac{l_{t}}{\bar{c}} \frac{d \varepsilon}{d \alpha}=C_{Z_{\dot{\alpha}}} \frac{l_{t}}{\bar{c}} \\
C_{m_{u}}=\frac{\partial C_{m}}{\partial M} M_{0}, \quad C_{m_{q}}=-2 \eta C_{L_{\alpha t}} V_{H} \frac{l_{t}}{\bar{c}}=C_{Z_{q}} \frac{l_{t}}{\bar{c}}, \quad C_{m_{\delta e}}=-\eta V_{H} \frac{d C_{L_{\alpha t}}}{d \delta_{e}}=C_{Z_{\delta e}} \frac{l_{t}}{\bar{c}}
\end{gathered}
$$

where $u_{0}$ and $M_{0}$ are the steady-state velocity and Mach number, $Q$ is the free-stream dynamic pressure, $A_{R}$ is the aspect ratio, $C_{D_{0}}$ and $C_{L_{0}}$ are the drag and lift coefficients at the steady state, $C_{L_{\alpha}}$ is the derivative of aircraft's lift coefficient with respect to the angle of attack $\alpha, X_{\delta_{e}}$ can be assumed to be zero, $C_{m_{\alpha f}}$ is the derivative of fuselage's moment coefficient with respect to $\alpha, C_{L_{\alpha t}}$ is the derivative of horizontal tail's lift coefficient with respect to the angle of attack, $\bar{c}$ is the mean aerodynamic chord, $l_{t}$ is the distance from the aircraft's gravity center to the aerodynamic center of horizontal tail, $V_{H}=l_{t} S_{t} / S \bar{c}$ is the horizontal tail volume coefficient ( $S$ and $S_{t}$ are the wing and horizontal tail areas), $d \varepsilon / d \alpha$ is the derivative the downwash angle with respect to the angle of attack, $\eta$ is the horizontal tail effective factor, $\tau$ is horizontal effectiveness parameter, $x_{c g}$ and $x_{a c}$ are the longitudinal positions of center of gravity and aerodynamic center, $m$ is the aircraft's weight, $I_{y}\left(\mathrm{~kg} . \mathrm{m}^{2}\right)$ is the mass moments of inertia about $y$.

The homogeneous solution to Eq. (3) can be obtained by assuming a solution of the form as

$$
x(t)=\hat{x} e^{i \omega t},
$$

where $\hat{x}$ is the complex amplitude of $x, \hat{x}=\left(\Delta \hat{u}, \Delta \hat{w}, \Delta \hat{\omega}_{y}, \Delta \hat{\theta}\right)^{T} ;(i \omega)$ is the Laplace variable with $i=\sqrt{-1}$ (imaginary number) and $\omega$ is the complex frequency. 
The control vector is written as

$$
\eta_{x}=\hat{\eta}_{x} e^{i \omega t} \quad \text { with } \quad \hat{\eta}_{x}=\left(\Delta \hat{\delta}_{e}, \Delta \hat{\delta}_{T}\right)^{T} .
$$

From Eq. (10), we have

$$
\dot{x}(t)=i \omega \hat{x} e^{i \omega t} \quad \text { or } \quad \hat{\dot{x}}(t)=i \omega \hat{x} .
$$

Replacing $(i \omega)$ by Laplace variable $s$

$$
x(s)=H_{\eta}(s) \eta_{x}(s)=-(A-i \omega I)^{-1} B \eta_{x}(s),
$$

$I$ is the identify matrix, $A$ and $B$ are the matrices defined from Eqs. (3) and (4).

$$
A=\left[\begin{array}{cccc}
X_{u} & X_{w} & 0 & -g \\
Z_{u} & Z_{w} & u_{0} & 0 \\
M_{u}+M_{\dot{w}} Z_{u} & M_{w}+M_{\dot{w}} Z_{w} & M_{q}+M_{\dot{w}} u_{0} & 0 \\
0 & 0 & 1 & 0
\end{array}\right], \quad B=\left[\begin{array}{cc}
X_{\delta_{e}} & 0 \\
Z_{\delta_{e}} & 0 \\
M_{\delta_{e}}+M_{\dot{w}} Z_{\delta_{e}} & 0 \\
0 & 0
\end{array}\right] .
$$

Solving Eq. (13) to obtain $x(s)$ and using the inverse Laplace transform to obtain $x(t)$ from which responses of $\Delta u, \Delta w, \Delta \omega_{y}, \Delta \theta$ are determined.

The complex frequency $\omega$ is determined from the state equation without control with the frequency equation as follows

$$
\operatorname{det}(A-i \omega I)=|A-i \omega I|=0 .
$$

Eq. (15) is a polynomial of degree 4 for $\omega$

$$
\omega_{k}=\omega_{0 k}+i \zeta_{k}, \quad(k=1 \div 4) .
$$

Values of the frequency $\omega_{0}$ and the damping ratio $\zeta$ indicate the flight quality of the aircraft.

\subsection{Comparison of results on longitudinal perturbation responses of the Navion air- craft}

Different aircrafts have different coefficients for matrix $A$ and matrix $B$. In order to verify the accuracy of computer program calculating the longitudinal stability with elevator deflection, we have calculated the stability of Navion aircraft at elevator deflection angle $\delta_{e}=10^{\circ}$. The Navion aircraft had all the parameters of aerodynamics and stability $[4,5,12]$. Parameters for longitudinal stability calculations of Navion aircraft are given in Tabs. 5-6.

Fig. 10 shows longitudinal perturbation responses of Navion aircraft calculated by present code (Fig. 10(a)) in comparison with results [5] (Fig. 10(b)). It was observed that responses of $\Delta u, \Delta w, \Delta \omega_{y}, \Delta \theta$ and $\Delta h$ were similar. With the elevator deflection angle of $\delta_{e}=10^{\circ}$, the Navion aircraft has been reduced to $1768 \mathrm{~m}$ of altitude with control times $t_{1}=300 \mathrm{~s}$. Thus, the present program of longitudinal stability can be used for aircrafts and the UAV that its aerodynamic forces were shown in Section 2.1.

By using present code, the eigenvalues obtained from the longitudinal state matrix of the Navion aircraft were

$$
\omega_{(1,2)}= \pm \omega_{0(1,2)}+i \zeta_{(1,2)}= \pm 0.214+0.017 i, \omega_{(3,4)}= \pm \omega_{0(3,4)}+i \zeta_{(3,4)}= \pm 2.59+2.5 i
$$




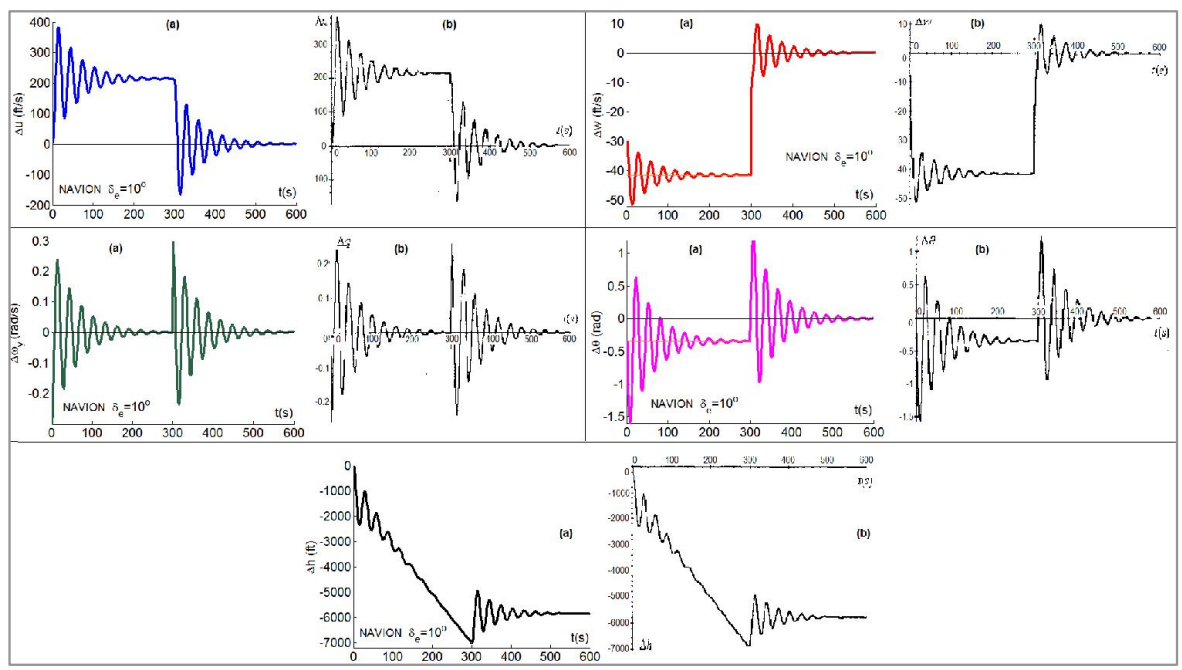

Fig. 10. Longitudinal perturbation responses of Navion aircraft (a) Present calculations; (b) Results [5]

In fact, oscillation only occurs with long period (with the frequency $\omega_{0}=0.214 \mathrm{~s}^{-1}$ and the damping ratio $\zeta=0.017 \mathrm{~s}^{-1}$ as shown in Fig. 10).

\subsection{Application for calculating longitudinal stability of the UAV with elevator deflec- tion}

The coefficients of matrix $A$ and $B$ are determined by the formulas (6)-(9). The value of each parameter of these formulas was determined by solving the aerodynamic problems for the UAV (Section 2.1) with the input values shown in Tabs. 5-6.

Table 5. Numerical values of the longitudinal stability parameters

\begin{tabular}{llcccccccc}
\hline Aircraft & $\begin{array}{c}U_{0} \\
(\mathrm{~m} / \mathrm{s})\end{array}$ & $\begin{array}{c}W \\
(\mathrm{~kg})\end{array}$ & $\begin{array}{c}\rho \\
\left(\mathrm{kg} / \mathrm{m}^{3}\right)\end{array}$ & $\begin{array}{c}I_{y} \\
\left(\mathrm{~kg} \cdot \mathrm{m}^{2}\right)\end{array}$ & $\begin{array}{c}S \\
\left(\mathrm{~m}^{2}\right)\end{array}$ & $\begin{array}{c}b \\
(\mathrm{~m})\end{array}$ & $\begin{array}{c}\bar{c} \\
(\mathrm{~m})\end{array}$ & $C_{D_{0}}$ & $R e$ \\
\hline Navion & 53.64 & 1247 & 1.225 & 4067 & 17.09 & 10.18 & 1.737 & 0.05 & $6.4 \times 10^{6}$ \\
UAV & 44.4 & 678 & 1.225 & 1350.4 & 12.94 & 15.4 & 0.875 & 0.034 & $2.6 \times 10^{6}$ \\
\hline
\end{tabular}

Table 6. Numerical values of the longitudinal stability parameters

\begin{tabular}{lllllllll}
\hline Aircraft & $C_{L_{0}}$ & $C_{D \alpha}$ & $C_{L \alpha}$ & $C_{m \alpha}$ & $C_{m \dot{\alpha}}$ & $C_{m q}$ & $C_{L \delta_{e}}$ & $C_{m \delta_{e}}$ \\
\hline Navion & 0.41 & 0.33 & 4.44 & -0.683 & -4.36 & -9.96 & 0.335 & -0.923 \\
UAV & 0.707 & 0.226 & 6.1 & -2.26 & -11.4 & -26.4 & 0.117 & -2.17 \\
\hline
\end{tabular}




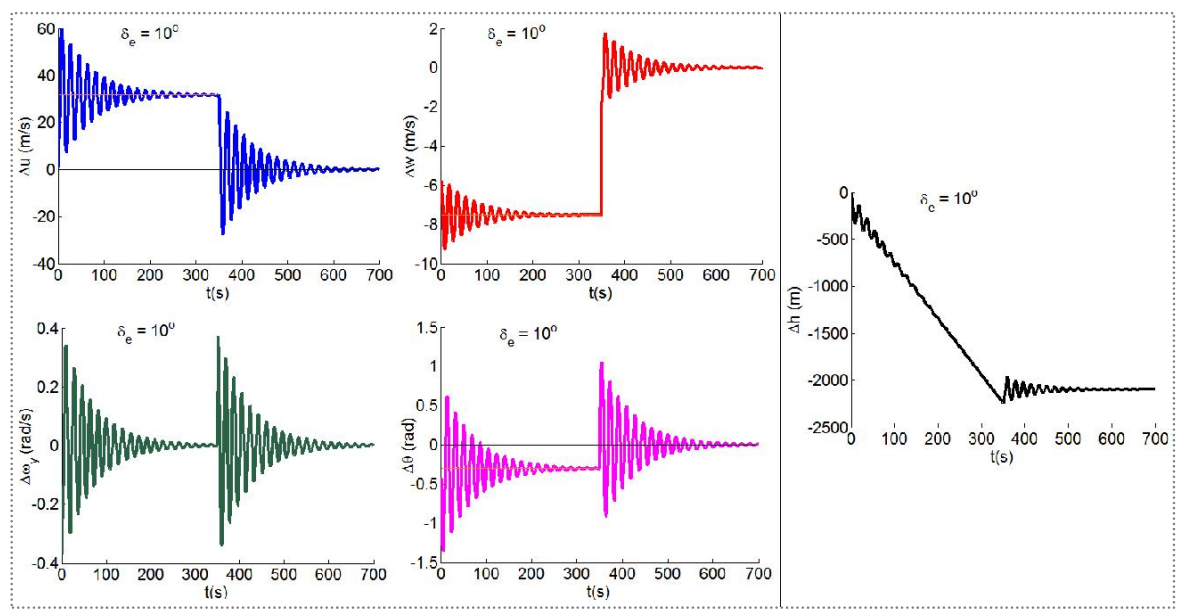

Fig. 11. Longitudinal perturbation responses of the $\mathrm{UAV}$ with $\delta_{e}=10^{\circ}$

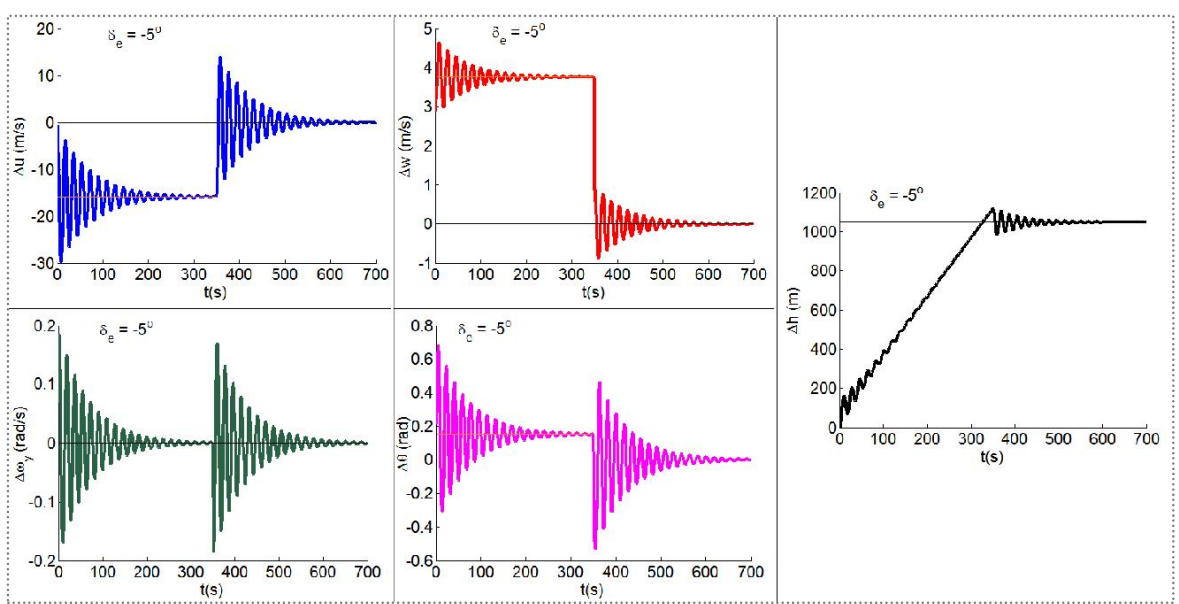

Fig. 12. Longitudinal perturbation responses of the UAV with $\delta_{e}=-5^{\circ}$

Figs. 11-12 present longitudinal perturbation responses of the UAV with elevator deflection angles $\delta_{e}=10^{\circ}$ and $\delta_{e}=-5^{\circ}$. At $t_{1}=350 \mathrm{~s}$, variables $\Delta u, \Delta w, \Delta \omega_{y}, \Delta \theta, \Delta h$ were convergent to certain values and the altitude changed $\Delta h=-2097 \mathrm{~m}$ (for $\delta_{e}=10^{\circ}$ ) and $\Delta h=1049 \mathrm{~m}$ (for $\delta_{e}=-5^{\circ}$ ) from the original altitude. However, these two values of $\Delta h$ did not get stability at the time $t_{1}=350 \mathrm{~s}$. It took time $t_{1}$ for the perturbation variables to be stabilized at new altitude when returning to $\delta_{e}=0^{\circ}$. Thus, this time $t_{1}$ was dependent on the aerodynamics and configuration of the aircraft. It might be reasonable, or too long (close to neutral stability), or indefinite (instability).

The eigenvalues obtained from the longitudinal state matrix of the UAV were

$\omega_{(1,2)}= \pm \omega_{0(1,2)}+i \zeta_{(1,2)}= \pm 0.345+0.0143 i, \omega_{(3,4)}= \pm \omega_{0(3,4)}+i \zeta_{(3,4)}= \pm 4.389+3.5 i$. 
Oscillations with the long period had frequency $\omega_{0}=0.345 \mathrm{~s}^{-1}$ and damping ratio $\zeta$ $=0.0143 \mathrm{~s}^{-1}$.

The numerical values of the frequency and damping ratio characterized longitudinal flying qualities of the UAV and Navion aircraft are shown in Tab. 7 (period $T=2 \pi / \omega_{0}$ ). Considering the values of $\omega_{0}$ and $\zeta$ of the long period mode, the Navion aircraft was stabilized a little faster than the UAV [13].

Table 7. Longitudinal flying qualities of the UAV and Navion aircraft

\begin{tabular}{lcccccc}
\hline \multirow{2}{*}{ Aircraft } & \multicolumn{3}{c}{ Long period (phugoid) } & \multicolumn{3}{c}{ Short period } \\
\cline { 2 - 7 } & $\omega\left(\mathrm{s}^{-1}\right)$ & $T(\mathrm{~s})$ & $\zeta\left(\mathrm{s}^{-1}\right)$ & $\omega\left(\mathrm{s}^{-1}\right)$ & $T(\mathrm{~s})$ & $\zeta\left(\mathrm{s}^{-1}\right)$ \\
\hline Navion & 0.214 & 29.36 & 0.017 & 2.59 & 2.42 & 2.5 \\
UAV & 0.345 & 18.2 & 0.014 & 4.39 & 1.8 & 3.5 \\
\hline
\end{tabular}

\section{CONCLUSIONS}

Our findings in this study have shown that in terms of aerodynamics, the contribution of the horizontal tail to the aircraft's lift and drag coefficients was not much. However, with the large distance from the aerodynamic center of the horizontal tail to the gravity center of the aircraft, the horizontal tail contributed a major part to the aircraft's pitching moment, which was a fundamental factor in equilibrium and longitudinal stability of the aircraft. A slight change in aerodynamic force on the horizontal tail can cause a significant change in the pitching moment of the aircraft. The horizontal tail is behind the main wing, so the calculation of the aerodynamic force on the horizontal tail is not as simple as a minor wing. Upstream flow of the main wing has uniform velocity at infinity. But for the horizontal tail, upstream flow of the tail is disturbed in the main wing wake, strongly influenced by main wing downward caused by effect of the wing tip. Thus, the method of calculating aerodynamic forces on the horizontal tail when considering the horizontal tail is a component of the aircraft was suitable. With intricate interferences between the wing and fuselage, between the wing and tail, phenomena of wing downwash, the choice of the method of solving the differential equations for viscous flows using Fluent software was reasonable. The dynamic stability problem mentioned in this study was to determine the longitudinal flying qualities of aircraft with elevator deflection corresponding to certain configuration and static margin of the aircraft. Good or bad flying qualities require feedback to adjust the aerodynamic dimensions of each component as well as the overall configuration of the aircraft.

\section{REFERENCES}

[1] L. Smith. Investigation of a modified low-drag body for an alternative wing-body-tail configuration. PhD thesis, University of Pretoria, South Africa, (2017).

[2] A. Paziresh, A. H. Nikseresht, and H. Moradi. Wing-body and vertical tail interference effects on downwash rate of the horizontal tail in subsonic flow. Journal of Aerospace Engineering, 30, (4), (2017). https://doi.org/10.1061/(asce)as.1943-5525.0000704. 
[3] D. F. Thomas Jr and W. D. Wolhart. Static longitudinal and lateral stability characteristics at low speed of 45 degree sweptback-midwing models having wings with an aspect ratio of 2, 4, or 6 . Technical note 4077, National advisory committee for aeronautics, (1957).

[4] R. C. Nelson. Flight stability and automatic control. McGraw-Hill Education, Inc., (1998).

[5] N. X. Hung. Dynamics and stability of airplane. Vietnam National University, Hanoi, (2004).

[6] J. Welstead. Conceptual design optimization of an augmented stability aircraft incorporating dynamic response performance constraints. PhD thesis, Auburn, Alabama, USA, (2014).

[7] M. Ghoreyshi, I. Greisz, A. Jirasek, and M. Satchell. Simulation and modeling of rigid aircraft aerodynamic responses to arbitrary gust distributions. Aerospace, 5, (2), (2018). https://doi.org/10.3390/aerospace5020043.

[8] D. Keller. Numerical approach aspects for the investigation of the longitudinal static stability of a transport aircraft with circulation control. In New results in numerical and experimental fluid mechanics IX, Springer, (2014), pp. 13-22. https://doi.org/10.1007/978-3-319-03158-3_2.

[9] H. T. B. Ngoc and N. M. Hung. Calculation of transonic flows around profiles with blunt and angled leading edges. Vietnam Journal of Mechanics, 38, (1), (2016), pp. 1-13. https://doi.org/10.15625/0866-7136/38/1/4177.

[10] H. T. B. Ngoc and N. M. Hung. Study of separation phenomenon in transonic flows produced by interaction between shock wave and boundary layer. Vietnam Journal of Mechanics, 33, (3), (2011), pp. 170-181. https://doi.org/10.15625/0866-7136/33/3/210.

[11] M. Mahdi. Prediction of wing downwash using CFD. In 3rd International Workshop on Numerical Modelling in Aerospace Sciences, Romania, Vol. 7, (2015), pp. 105-111.

[12] E. Seckel and J. J. Morris. The stability derivatives of the Navion aircraft estimated by various methods and derived from flight test data. National Technical Information Service, USA, (1971).

[13] S. Paudel, S. Rana, S. Ghimire, K. K. Subedi, and S. Bhattrai. Aerodynamic and stability analysis of blended wing body aircraft. International Journal of Mechanical Engineering and Applications, 4, (4), (2016), pp. 143-151. https://doi.org/10.11648/j.ijmea.20160404.12. 